\title{
Imaging of optic nerve head drusen with the scanning laser ophthalmoscope
}

\author{
R J Haynes, A Manivannan, S Walker, P F Sharp, J V Forrester
}

\begin{abstract}
Background-Optic nerve head drusen may present diagnostic difficulties in cases of disc swelling. Imaging of the nerve in a search for drusen is often inconclusive, especially in children, where drusen may be buried below the surface of the nerve head.

Methods-A small study was carried out using a scanning laser ophthalmoscope (SLO) with an infrared confocal facility to scan deep within optic discs in an attempt to image drusen.

Results-The SLO was able to demonstrate superficial and buried drusen (using the infrared confocal facility). The superiority of the SLO over ultrasound in the presence of lens opacity was revealed, as the SLO simultaneously demonstrated both drusen and the associated anomalous disc features which are not detected by ultrasound.

Conclusion-The SLO can help in the diagnosis of optic disc drusen especially in difficult cases where lens opacity or buried drusen hinders their definitive diagnosis. (Br f Ophthalmol 1997;81:654-657)
\end{abstract}

Imaging of optic nerve head drusen has been attempted by several different techniques, with varying degrees of success. Recent work has shown that fluorescein angiography may show autofluorescence and late staining of disc drusen in some cases; that computed tomography (CT) and magnetic resonance imaging (MRI) inconsistently show superficial calcification or lucency respectively; and that only B-mode ultrasonography consistently demonstrates calcified optic disc drusen. ${ }^{1}$ However, few of the associated diagnostic features of discs with drusen are demonstrated by ultrasound and in the presence of opacity of the media, doubt over the possibility of dual pathology will persist. In this brief report, we demonstrate the value of the scanning laser ophthalmoscope (SLO) in demonstrating optic disc drusen and the associated disc abnormalities, even in the presence of lens opacity.

\section{Materials and methods}

SLO imaging of 12 eyes with optic nerve head swelling was carried out using a custom built device. The construction and performance characteristics of this instrument are detailed elsewhere. ${ }^{2}$ Briefly, the SLO uses a highly col- limated narrow beam of laser light to sweep over the retina, delivering all its energy to a very small spot for a very short time. Light returning from that spot is detected and synchronously decoded to form an image on a monitor. The SLO has 'confocal optics', meaning that detection of returning light takes place at a focal point which is conjugate to the focus of the illumination spot on the retina. This is achieved by the use of a confocal aperture which allows only light which is brought to a focal point at the aperture to pass through the aperture and thus to the detector. Any light brought to focus in front of, or behind the aperture (that is, light arising from a focal plane deep to, or superficial to the confocal point) will not pass through the aperture and will not be detected.

Therefore, the SLO allows analysis of light from a particular depth of the illuminated fundus. The slice thickness analysed is dependent on the size of the confocal aperture; a smaller aperture results in a thinner slice, but also a weaker signal as less light returns to the detector. In this study a $200 \mu \mathrm{m}$ confocal aperture was used.

An infrared diode laser (780 $\mathrm{nm})$ and a visible dye laser $(540 \mathrm{~nm})$ were used for illumination, at an incident power at the cornea of $200 \mu \mathrm{W} / \mathrm{cm}^{2}$. Confocal and indirect images were captured. Indirect mode permits only the passage of scattered light back to the detector and, therefore, removes any directly reflected light. Suitable images were grabbed and stored on a PC compatible computer (Elonex PC450). The SLO images were of a $25^{\circ}$ field and at a resolution of $512 \times 512$ pixels. Binocular indirect ophthalmoscopy, B-mode ultrasound scan, and automated visual field analysis were performed on all patients. Fundus photographs were taken for comparison. All investigations were carried out with the informed consent of the patients.

\section{Results}

Binocular indirect ophthalmoscopy and fundus photography allowed imaging of superficial drusen in the presence of clear ocular media (Fig 1(A)). The SLO short wavelength (540 $\mathrm{nm}$, green) light produced high resolution images of superficial disc drusen and other superficial structures such as the retinal vessels and the nerve fibre layer (Fig 1(B)). Long wavelength (780 nm, infrared) light using the confocal mode allowed identification of buried drusen (black arrows) and other deep struc- 

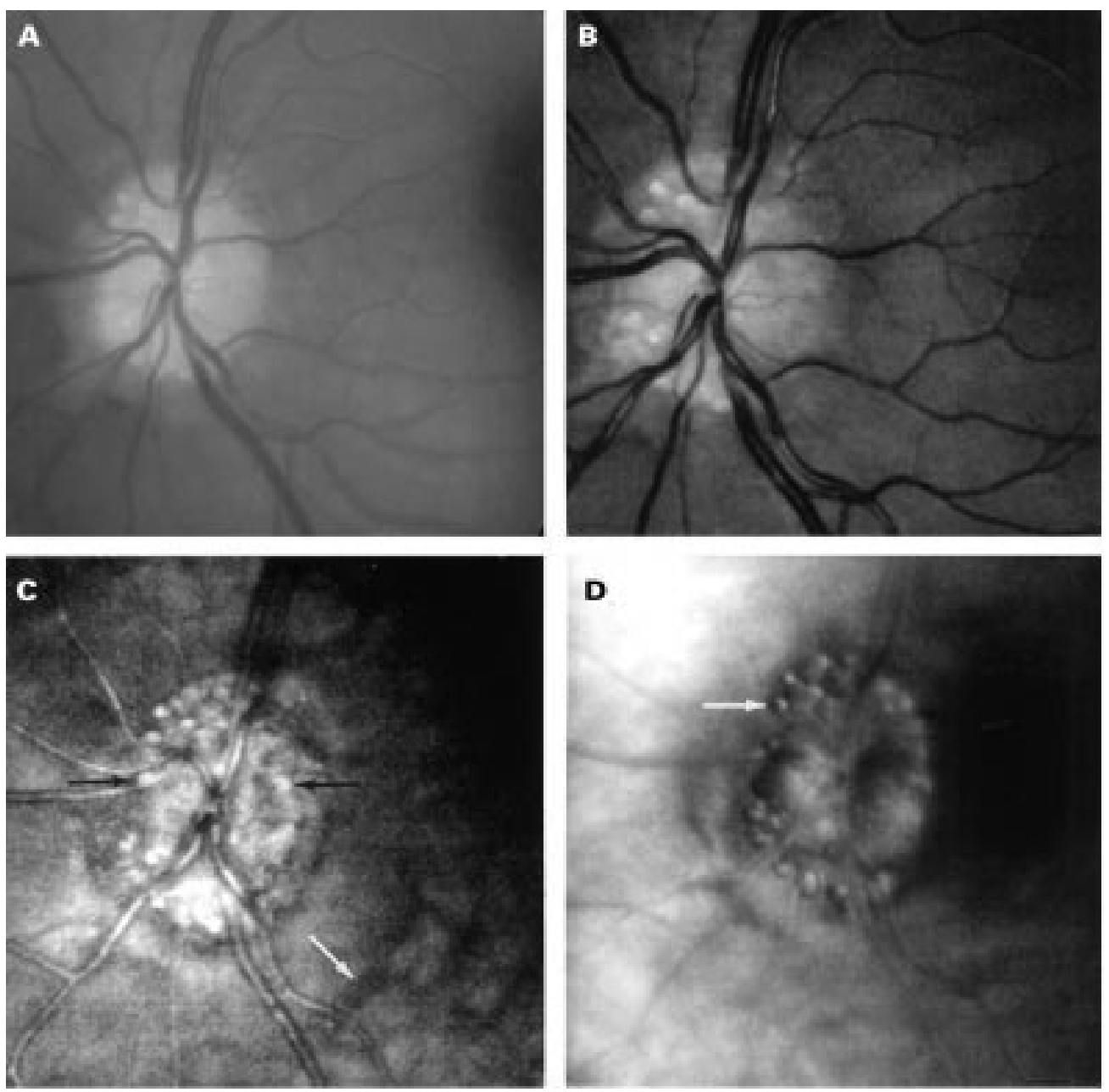

Figure 1 Fundus images of a patient with optic disc drusen. (A) Fundus photograph showing superficial disc drusen. (B) Short wavelength (540 nm, green) SLO images showing superficial structures including disc drusen, retinal vessels, and the nerve fibre layer. (C) Long wavelength ( $780 \mathrm{~nm}$, infrared) confocal mode SLO images showing more drusen including buried ones (black arrows) which are not visible in $(A)$ or $(B)$. Note the retinal vessels appear out of focus as they lie superficial to the plane of focus, whereas deep choroidal vessels are now visible (white arrow). (D) Indirect mode (780 nm, infrared) SLO images of disc drusen showing the distinctive 'bubble' appearance.

tures such as choroidal vessels (white arrow) (Fig 1(C)). Note the retinal vessels appear out of focus as they lie superficial to the plane of focus.

Indirect mode (780 nm, infrared) images of disc drusen produced a distinctive 'bubble' appearance (Fig 1(D)), which correlates well with the appearance of macular drusen imaged with the indirect mode SLO. ${ }^{45}$ Drusen are detected by the indirect mode SLO because they scatter a large amount of light; indirect mode imaging utilises mainly scattered light.

The distinctive indirect mode 'bubble' appearance of drusen is due to the fact that the convex surface of the drusen facing the eccentrically placed confocal aperture will preferentially scatter light towards the aperture when illuminated; this surface of the drusen will thus appear light. When the scanning beam moves to the opposite side of the drusen surface (which faces away from the confocal aperture) less light will be scattered towards the aperture, and this side of the drusen will thus appear darker. The appearance of brightness on one side and darkness on the other side of the drusen results in the optical impression of a convex bubble.
Optic disc drusen were demonstrated in 10 of the 12 eyes examined with the SLO and B-mode ultrasound; image details are listed in Table 1. One of the patients had significant lens opacities, and disc drusen could not be visualised by either binocular indirect ophthalmoscopy or fundus photography (Fig 2 (A)). However, the SLO was able to generate good contrast images of disc drusen in this patient and secured the diagnosis (Fig 2 (B)). The clinical impression in the sixth patient of crowded discs without drusen, was confirmed by their absence on the confocal SLO and B-mode ultrasound.

Automated perimetry confirmed the presence of field defects in all of our cases of disc drusen. Concordance between the site of the disc drusen (and therefore loss of axons) and the spatial location of the field defects was found in some but not all patients (Table 1). This may be due to a global impairment of optic nerve function in some eyes with disc drusen, or poor performance in the automated perimetry tests.

Other features of disc swelling due to drusen demonstrated by the SLO included anomalous branching of retinal vessels; absent optic cup; 

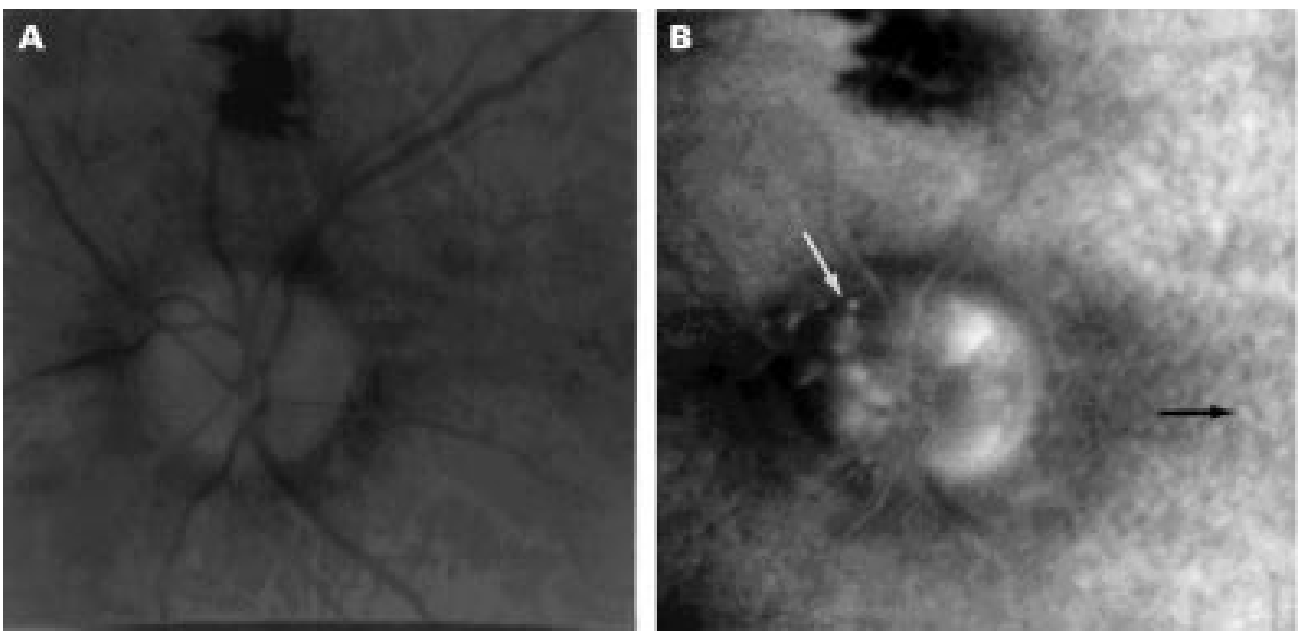

Figure 2 (A) Fundus photograph showing the poor view of disc drusen in a patient with significant lens opacity. (B) Indirect mode SLO image of the same patient demonstrating disc drusen (white arrow) and retinal drusen (black arrow) in the presence of lens opacity.

Table 1 Summary of results

\begin{tabular}{|c|c|c|c|c|c|c|c|}
\hline Age & Sex & Symptoms & Eye & Signs & SLO & Fields & Ultrasound \\
\hline \multirow[t]{2}{*}{36} & $\mathrm{~F}$ & $\begin{array}{l}\text { Blurring of vision } \\
\text { during pregnancy }\end{array}$ & $\mathrm{R}$ & Drusen & $\begin{array}{l}\text { Drusen } \\
\text { temporally }\end{array}$ & Nasal defects & + \\
\hline & & & $\mathrm{L}$ & Drusen + L RAPD & Drusen +++ & Diffuse defects & + \\
\hline \multirow[t]{2}{*}{83} & $\mathrm{~F}$ & Decreased vision & $\mathrm{R}$ & $\begin{array}{l}\text { No drusen visible due } \\
\text { to cataract }\end{array}$ & Diffuse drusen & Diffuse defects & + \\
\hline & & & $\mathrm{L}$ & $\begin{array}{l}\text { No drusen visible due } \\
\text { to cataract }\end{array}$ & Superior drusen & $\begin{array}{l}\text { Inferior nasal } \\
\text { step }\end{array}$ & + \\
\hline \multirow[t]{2}{*}{42} & $M$ & None & $\mathrm{R}$ & Drusen & Superior drusen & Constricted & + \\
\hline & & & $\mathrm{L}$ & Drusen & Nasal drusen & Inferior arcuate & + \\
\hline \multirow[t]{2}{*}{18} & $\mathrm{~F}$ & None ${ }^{\star}$ & $\mathrm{R}$ & Drusen & Diffuse drusen & Constricted & + \\
\hline & & & $\mathrm{L}$ & Drusen & Diffuse drusen & Constricted & + \\
\hline \multirow[t]{2}{*}{18} & $\mathrm{~F}$ & Poor vision on $\mathrm{R}^{\star}$ & $\mathrm{R}$ & Drusen & $\begin{array}{l}\text { Macular bundle } \\
\text { drusen+ }\end{array}$ & $\begin{array}{l}\text { Marked } \\
\text { constriction }\end{array}$ & + \\
\hline & & & $\mathrm{L}$ & Drusen & Diffuse drusen & Constricted & + \\
\hline \multirow[t]{2}{*}{17} & $\mathrm{~F}$ & Headache & $\mathrm{R}$ & Swollen disc? & No drusen & Normal & - \\
\hline & & & $\mathrm{L}$ & Swollen disc? & No drusen & Normal & - \\
\hline
\end{tabular}

^Normal CT/MRI scan.

spontaneous venous pulsation; irregular disc edge with retinal pigment epithelial disturbance; and the absence of capillary telangiectasia, cotton wool spots, peripapillary retinal folds, and haemorrhages that would be suggestive of other causes of disc swelling.

\section{Discussion}

Histopathologically, optic nerve drusen are calcified, intracellular mitochondria of optic nerve axons. ${ }^{6}$ They are present clinically in $0.3 \%$ of individuals and histopathologically in $2 \%$ of individuals, being present almost exclusively in white patients and found bilaterally in $75 \%$. $^{7}$ Inheritance may be autosomal dominant with variable penetrance and there is an association with retinitis pigmentosa and angioid streaks. ${ }^{8}$ Visual field defects affect up to $87 \%$ of patients, ${ }^{9}$ all of our confirmed cases of disc drusen having field loss. Subretinal neovascular membranes and haemorrhages may also occur.

In this study the SLO has shown itself to be an excellent method for the diagnosis of optic disc drusen and its associated nerve head abnormalities even in the presence of significant lens opacity. B-mode ultrasonography has also been shown to be effective for demonstration of calcified disc drusen; however, the asso- ciated optic nerve features are not demonstrated simultaneously by ultrasound and in the presence of media opacity, dual pathology would be difficult to exclude conclusively. Recent experience of confocal mode SLO imaging of macular drusen with visible wavelengths in our department has shown considerably superior image contrast compared with fundus photographs. ${ }^{5}$ Confocal SLO disc drusen images may therefore be suitable for quantitative image analysis, as the area of the disc affected by drusen theoretically may correlate with the visual field deficit. Longitudinal studies may also point to parallel changes in drusen and fields over time. These are a potential areas of future investigation.

We acknowledge Scotia Pharmaceuticals and the Wellcome Trust for supporting this research. Also we thank Miss D Campbell for photographic assistance.

1 Kheterpal S, Good P, Beale D, Kritzinger E. Imaging of optic disc drusen: a comparative study. Eye 1995;9:67-9.

2 Manivannan A, Sharp PF, Phillips RP, Forrester JV. Digital fundus imaging using a scanning laser ophthalmoscope. Physiol Meas 1993;14:43-56.

3 Manivannan A, Sharp PF, Forrester JV. Performance measurements of an infrared digital scanning laser ophthalmourements of an infrared digital scan

4 Manivannan A, Kirkpatrick JNP, Sharp PF, Forrester JV. Clinical investigation of an infrared digital scanning laser Clinical investigation of an infrared digital scanning
ophthalmoscope. Br f Ophthalmol 1994;78:84-90. 
5 Kirkpatrick JNP, Spencer T, Manivannan A, Sharp PF, Forrester JV. Quantitative image analysis of macular drusen
from fundus photographs and scanning laser ophthalmofrom fundus photographs and scant
scope images. Eye 1995;9:48-55.

6 Folberg R, Campbell RJ, Luckenbach M, Margo CE, Proia $\mathrm{AD}$, Schachat A. Ophthalmic pathology and intraocular tumours. San Francisco: American Academy of Ophthalmology Basic and Clinical Science Course 1994-1995, Section $4: 176$.
7 Rosenberg MA, Savino PJ, Glaser JS. A clinical analysis of pseudopapilledema. I Population, laterality, acuity, refractive error, ophthalmoscopic characteristics, and coincident disease. Arch Ophthalmol 1979;97:71-5.

8 Boye SW, Platia EV, Green WR. Drusen of the optic nerve head. Ann Ophthalmol 1978;10:695-704.

9 Cohen D. Drusen of the optic disc and the development of field defects. Arch Ophthalmol 1971;85:224. 